\title{
Design, Analysis and Experimental Eerification of a Epatial Constant Force End-Effector for Polishing/Deburring Operations
}

\section{Bingxiao Ding}

Jishou University

Jixu Zhao

Jishou University,

Yangmin Li ( $\nabla$ yangmin.li@polyu.edu.hk)

The Hong Kong Polytechnic University https://orcid.org/0000-0002-4448-3310

\section{Research Article}

Keywords: Compliant Mechanism, Constant Force Mechanism, Polishing/deburring Operation.

Posted Date: May 24th, 2021

DOl: https://doi.org/10.21203/rs.3.rs-455800/v1

License: (c) (i) This work is licensed under a Creative Commons Attribution 4.0 International License.

Read Full License

Version of Record: A version of this preprint was published at The International Journal of Advanced Manufacturing Technology on July 21st, 2021. See the published version at https://doi.org/10.1007/s00170-021-07579-1. 


\title{
Design, analysis and experimental verification of a spatial constant-force end-effector for polishing/deburring operations
}

\author{
Bingxiao Ding ${ }^{1} \cdot$ Jixu Zhao ${ }^{1} \dagger \cdot$ Yangmin $\mathrm{Li}^{2} \dagger$
}

Received: 22/04/2021 / Accepted: date

\begin{abstract}
Controlling the contact force on workpieces is a challenging task for industrial deburring operations. To solve this issue, a novel constant force mechanism(CFM) based on the combination of positive and negative stiffness mechanism is proposed by using folding beam and bi-stable beam. Without using any additional sensors and control algorithms, the proposed mechanism can produce a travel range in constant force manner. In this paper, the design concepts, analytical modeling, finite element analysis (FEA) simulation and experimental studies are presented and discussed. Firstly, a novel spatial CFM is proposed and using the pseudo rigid body $(\mathrm{PRB})$ to established the mathematical model of the whole mechanism. Then, the FEA simulation is performed to validate the correctness of theoretical analysis. In addition, to eliminate the force variation, particle swarm optimization (PSO) method is utilized to find optimal architectural parameters solutions of the CFM. Finally, the experimental tests are performed to verify the performance of the designed CFM. The configuration design and parameter optimization can be further applied to the design of other CFM mechanisms for polishing operations.
\end{abstract}

Keywords Compliant Mechanism - Constant Force Mechanism · Polishing/deburring Operation .

Jixu Zhao; Yangmin Li zjy13575154725@hotmail.com;yangmin.li@polyu.edu.hk 1

School of Physics and Electromechanical Engineering, Jishou University, Jishou, Hunan, 416000, China 2

Department of Industrial and Systems Engineering, The Hong Kong Polytechnic University, Hong Kong SAR, 999077, China

\section{Introduction}

Robots are increasingly used for workpieces deburring and polishing to overcome the problems of low production efficiency, high cost and poor consistency in manual polishing[1]-[3]. As pointed in previous research, the contact force between the grinding tool and work-piece is the key factor that affects the polishing quality $[4,5]$. Excessive or insufficient contact force would greatly impair the quality of the surface roughness. Thus, to maintain the constant contact force in the grinding process to ensure the polishing quality and consistency becomes an urgent issue [6]-[8] .

Generally speaking, the constant force output can be realized by two methods[9]. The first way is an active compliance force control. The polishing force can be controlled by a closed-loop controller. In addition, the robot can actively adapt to the changing of the machined surface to ensure the constant contact force[10, 11]. Masoud et al proposed a resultant force control strategy for light abrasive surface grinding. The performance of resultant force control strategy with real-time TDC algorithm is validated by the grinding experimental studies[12]. Wang et al designed a vibration suppression method for large thin-walled shell grinding based on force control algorithm. The experimental results show that the grinding depth error is less than $\pm 0.1 \mathrm{~mm}$, and the polishing quality of the workpiece surface can be significantly improved [13]. Xie proposed a method for grinding blade free-form surface based on PSO algorithm. The impedance controller based on PSO algorithm can realize the function of impedance parameters adjustment and the contact force between workpiece and grinding tool can be accurately controlled[14]. Xu put forward a zero drift and gravity compensation algorithm for robot belt grinding with complex geometry 
which combines force-position hybrid control strategy with PI controller to maintain the stability of force control in robot grinding process[15]. Although constant contact force can be realized by aforementioned methods, needing complex controller algorithm and expensive sensors limits their further applications. Moreover, the positioning accuracy will degrade the force control performance through the force-position hybrid controller to realize active compliance force control[16].

In contrast, another method is the passive compliance force control which uses rigidity of the robot and the flexibility of the force controllable end-effector to realize force control. The path planning is realized by the robotic arm and the control of force is realized by the end-effector respectively[17,18]. Mohammad proposed a force controllable end-effector for automatic deburring, which can reduce the inertial effect of polishing motor and spindle driven by micro driver[19]. Chen proposed an end-effector, including gravity compensation force controller and two new eddy current dampers, which were integrated into the end-effector to improve the dynamic performance of the system and suppress the vibration during grinding and polishing[20]. Liu proposed a new polishing mechanism, and detailed modeling and analysis of hardware circuit structure and force control algorithm design, The polishing actuator has good compliance performance[21]. Tang proposed a multi degree of freedom flexible terminal actuator for mould polishing robot. The buffer spring ensures the working pressure between the grinding head and the workpiece changes in a constant range [22]. The key component of the force controllable end-effector is the constant force compensation device. At present, the commonly used constant force compensation devices are type of electromagnetic, air bag and linear motor[23].

However, the above constant force compensation devices possess the disadvantages of low precision, slow response and poor environmental adaptability. In view of these shortcomings, a novel spatial CFM for grinding operation based on distributed flexure beam is proposed in this paper. The remainder of this paper is organized as follows: the design concepts of proposed CFM is depicted in Section 2; Section 3 establishes the mathematical model of the whole CFM and obtains the relationship between output force and input displacement; ANSYS Workbench is utilized to verify correctness of the theoretical analysis in Section 4; To eliminate the force variation the PSO method is adopted to optimize the CFM in Section 5; In addition, experimental studies are conducted in Section 6 to verify the performance of the CFM; Finally, conclusions are made in Section 7.

\section{Design Concepts}

CFM is a passive constant force system, which can provide a constant output force within a certain range of deformation relying on the characteristics of the structure itself. Compared with the active constant force system, the CFM can reduce the use of expensive sensors and complex control algorithms, thus reducing the cost.

To realize a constant force output, the combination of positive stiffness mechanism and negative stiffness mechanism is a typical method. Generally, the straight beam is taken as positive stiffness mechanism to neutralize negative stiffness region of bi-stable beam mechanism. However, the traditional symmetrical straight beam mechanism cannot take full advantage of negative stiffness region of the bi-stable beam mechanism. To overcome this issue, a novel type of folding beam as a positive stiffness mechanism is designed for the purpose of increasing the travel range constant force output. The force-displacement relationship comparison of designed positive stiffness mechanism and traditional mechanism is depicted in Fig.1 which shows that the folded beam mechanism has a good linearity in long range.

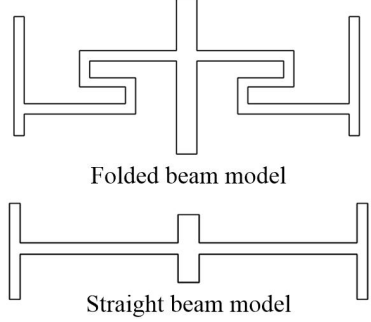

(a)

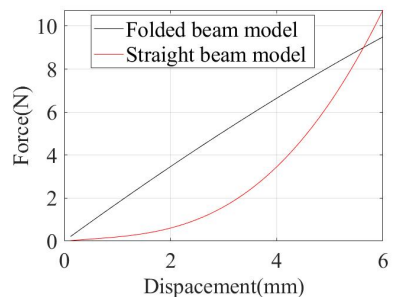

(b)
Fig. 1 Comparison of two kinds of positive stiffness mechanism.

The autonomous polishing scenario using CFM system is shown in Fig.2, the end-effector including voice coil motor, CFM and grinding head are installed in the end of robot arm through the connecting flange. When the voice coil motor works, it drives the grinding head to approach the workpiece. As an intermediate part, the CFM placed between the motor unit and grinding tool and the grinding tool mounted on the CFM. Due to the constant force output characteristics of the CFM, the grinding head can maintain a constant force contact with the workpiece in a certain range of motion, so as to finish the precision grinding and polishing. 

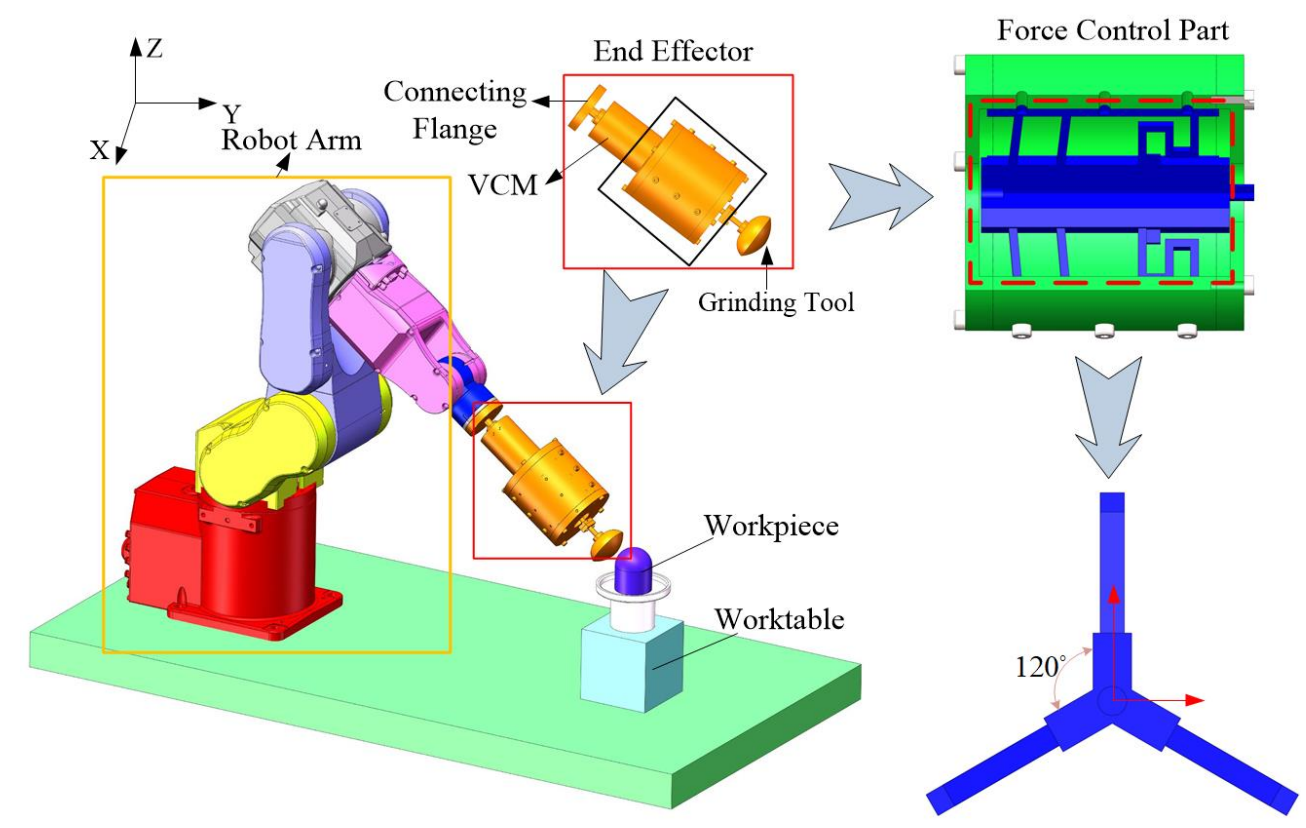

Fig. 2 Structure design of grinding mechanism with constant output force.

\section{Analytical design model}

In this part, the analytical model of bi-stable beam and folding beam is established respectively, then, the relationship between input force and output displacement of the CFM is derived. As shown in Fig.2, the CFM is composed of three branched chains and a central column, each chain composed of a parallel bi-stable beams and a folding beam mechanism. Considering the identical architecture of each branch, an arbitrary chain is selected for analysis.

\subsection{Modeling of bi-stable beam mechanism}

As shown in Fig.3, buckling and deformation of the bistable beam is resulted by the loading force $F_{n}$. In this figure, $L$ denotes the length of the beam, $\theta$ represents the inclination angle between the bi-stable beam and the $X$ axis, and $\Delta x_{b}$ is the displacement of the bistable beam under the force of $F_{n}$ along $Y$ direction.

When the force $F_{n}$ loading on the bistable beam exceeds the critical force of the material, referring to the previous studies the buckling force of the $i$ critical point of the guide beam can be written as $[24,25]$ :

$F_{n}=\frac{4 E I\left(\lambda_{n}\right)^{2}}{L^{2}}$

where $\lambda_{n}=\pi, 4.493,2 \pi, \ldots, n=1,2,3, \ldots$

The negative stiffness of bistable beam $k_{1}$ can be expressed as follow:

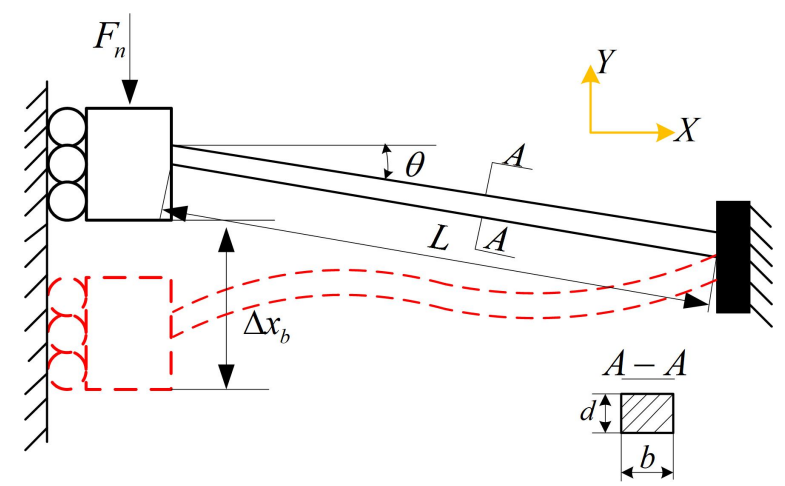

Fig. 3 Buckling deformation of bistable beam mechanism.

$k_{1} \approx \frac{33 E I}{L^{3}}$

Where $E$ represents the elastic modulus of the material, $I=b d^{3} / 12$ is the section moment of inertia of the guide beam, $d$ is the thickness of the beam, $b$ is the width of the beam. Referring to equations (1) and (2), the force-displacement relationship of the bistable beam can be written as:

$F_{n}=E S \frac{\Delta x_{b}}{L}\left(\frac{\Delta x_{b}}{L}-\sin \theta\right)\left(\frac{\Delta x_{b}}{L}-2 \sin \theta\right)$

Where $S=b \cdot d$ is the cross sectional area of the guide beam. 


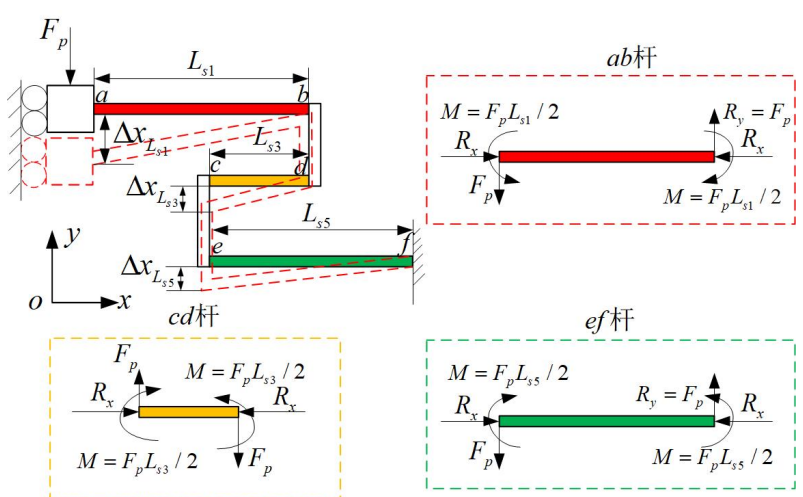

Fig. 4 Resolved components of folded beam.

\subsection{Modeling of folding beam mechanism}

The deformation of the folded beam is shown in Fig. 4 , in which $L_{s 1}, L_{s 3}$ and $L_{s 5}$ represent the length of each beam respectively, and $\Delta x_{f}$ is the displacement of the folded beam due to the external force $F_{p}$. The folding beam mechanism can be decomposed of three cantilevers $a b, c d$ and $e f$, and the deformation of each cantilever is $\Delta x_{L_{s 1}}, \Delta x_{L_{s 3}}$ and $\Delta x_{L_{s 5}}$ respectively. According to the superposition principle, the total deformation $\Delta x_{f}$ of the folding beam mechanism and stiffness $k_{2}$ of mechanism can be derived as following[26]:

$\Delta x_{f}=\Delta x_{L_{s 1}}+\Delta x_{L_{s 3}}+\Delta x_{L_{s 5}}$

$\frac{1}{k_{2}}=\frac{1}{k_{a b}}+\frac{1}{k_{c d}}+\frac{1}{k_{e f}}$

$a b, c d$ and ef beams can be regarded as semi-fixed beam model under concentrated load $2 F_{p}$, as shown in Fig. 5.

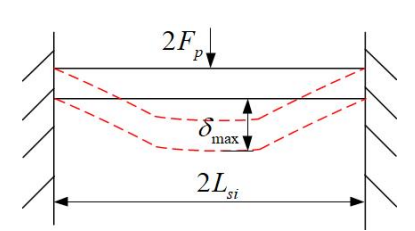

(a)

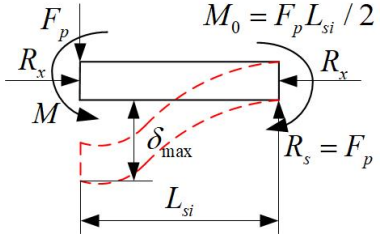

(b)
Fig. 5 (a) fixed-fixed beam under transverse loading (b) the model of half-fixed beam

In Fig.5(a), the length of the fixed beam is $2 L_{s i}$, and the middle of the beam is subjected to a downward concentrated load $2 F_{p}$. Under the force of $2 F_{p}$, the maximum deformation of the beam is $\delta_{\max }$. If the fixed beam is spilt in the middle, a semi-fixed beam can be obtained as shown in Fig.5(b). The bending moment of the semi-fixed beam at the fixed end is:

$M_{o}=\frac{F_{p} L_{s i}}{2}$

The shear force is:

$R_{s}=F_{p}$

The axial force can be neglected because it is too small compared with shear force and bending moment.

In a semi-fixed beam, the maximum displacement $\delta_{\max }$ is composed of the displacement $\delta_{M 0}$ caused by the bending moment $M_{0}$ and the displacement $\delta_{s}$ caused by the shear force $R_{s}$.

$\delta_{\max }=\delta_{M_{0}}+\delta_{s}$

According to Hooke's Law:

$\frac{1}{k_{\max }}=\frac{1}{k_{M_{0}}}+\frac{1}{k_{s}}$

For a semi-fixed beam, because of bending moment the maximum deflection occurs in the middle of the beam:

$\delta_{M_{0}}=\frac{F_{p} L_{s i}^{3}}{12 E I}$

Therefore, the stiffness constant caused by the bending moment of the fixed beam can be expressed as:

$k_{M_{0}}=\frac{12 E I}{L_{s i}^{3}}$

Where $L_{s i}$ is the length of the semi fixed beam. For semi fixed beams, the stiffness constant caused by bending moment is half of that of fixed beams. For semi fixed beams, the maximum deflection due to shear force is:

$\delta_{s}=\frac{6 F_{s} L_{s i}}{5 A G}$

where $G$ is the shear modulus, $G=E / 2(1+\mu), \mu$ is the Poisson's ratio, and $A$ is the area of the beam cross section. According to known conditions, $F_{s}=F_{p} / 2$, the results are as follows:

$\delta_{s}=\frac{6}{5} \frac{(1+\mu) F_{s} L_{s i}}{A E}$

The stiffness constant is:

$k_{s}=\frac{5}{6} \frac{A E}{(1+\mu) L_{s i}}$

By adding the above formula into (5), we can get the following results:

$$
\begin{aligned}
& \frac{1}{k_{2}}=\frac{L_{s 1}^{3}+L_{s 3}^{3}+L_{s 5}^{3}}{12 E I}+ \\
& \quad \frac{6\left(L_{s 1}+L_{s 3}+L_{s 5}\right)+6 \mu\left(L_{s 1}+L_{s 3}+L_{s 5}\right)}{5 E A}
\end{aligned}
$$


The relationship between the output force $F_{p}$ and the input displacement $\Delta x_{f}$ of the folded beam can be obtained by combining the above formula.

$F_{p}=k_{2} \Delta x_{f}$

When a branch chain of the CFM is subjected to an external force, the deformation of the bistable beam equals to the deformation of the folded beam, namely $\Delta x_{b}=\Delta x_{f}$. By adding the above formula into (3) and (16), the relationship between the force of a branch chain $F_{1}$ and the input displacement $\Delta x$ can be obtained:

$$
\begin{aligned}
& F_{1}=F_{n}+F_{p} \\
& =E S \frac{\Delta x}{L}\left(\frac{\Delta x}{L}-\sin \theta\right)\left(\frac{\Delta x}{L}-2 \sin \theta\right)+\left(\frac{L_{s 1}^{3}+L_{s 3}^{3}+L_{s 5}^{3}}{12 E I}\right. \\
& \left.+\frac{6\left(L_{s 1}+L_{s 3}+L_{s 5}\right)+6 \mu\left(L_{s 1}+L_{s 3}+L_{s 5}\right)}{5 E A}\right)^{-1} \Delta_{x}
\end{aligned}
$$

\section{FEA verification}

In this section, the FEA simulation is utilized to verify the correctness of the theoretical analysis. The adopted material is PLA-ST. The material properties are with Young modulus 1477MPa, Poisson's ratio 0.3, and the density $1.25 \mathrm{~g} / \mathrm{cm}^{3}$. The original dimensional parameters of the designed CFM is listed in Table 1.

Table 1 Original architecture parameters of the CFM.

\begin{tabular}{lll}
\hline \hline parameter & value & unit \\
\hline $\mathrm{L}$ & 30 & $\mathrm{~mm}$ \\
$\mathrm{~b}$ & 1.0 & $\mathrm{~mm}$ \\
$\mathrm{~d}$ & 10 & $\mathrm{~mm}$ \\
$\theta$ & 5.0 & $\mathrm{deg}$ \\
$L_{s 1}$ & 18 & $\mathrm{~mm}$ \\
$L_{s 3}$ & 5 & $\mathrm{~mm}$ \\
$L_{s 5}$ & 18 & $\mathrm{~mm}$ \\
\hline \hline
\end{tabular}

The ANSYS Workbench is adopted to verify the performance of bistable and folding beams and results are depicted in Fig.6. Referring to the Fig.6(a), the critical buckling force point occurs at $(1.3,8.46)$ in theoretical which very closes to the point of $(1.2,9.99)$ in FEA results. At the same point in folded beam mechanism, the force value $2.86 \mathrm{~N}$ is also closed to the $3.09 \mathrm{~N}$. In addition, the slope of both negative stiffness and positive stiffness in theoretical and FEA simulation are very consistent.

Referring to Fig.7 It can be concluded that theoretical and simulation results are both present constant force characteristic and the trends of the two curves are

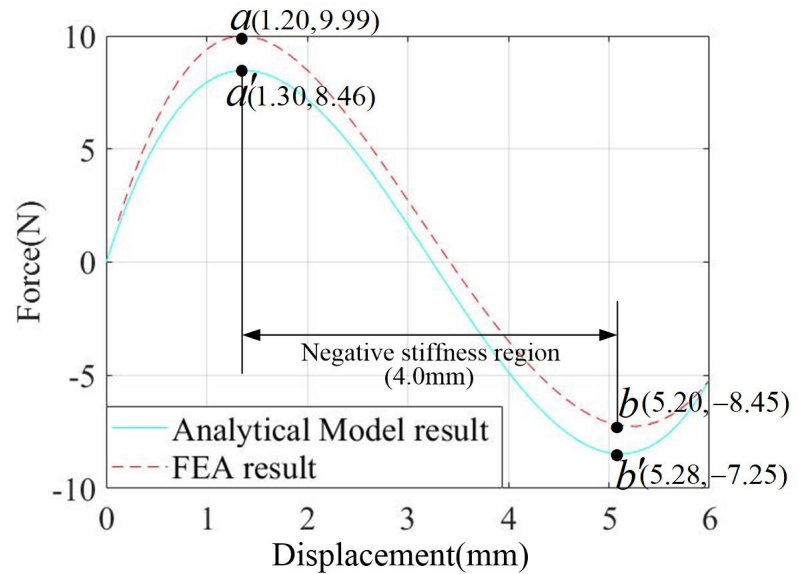

(a)

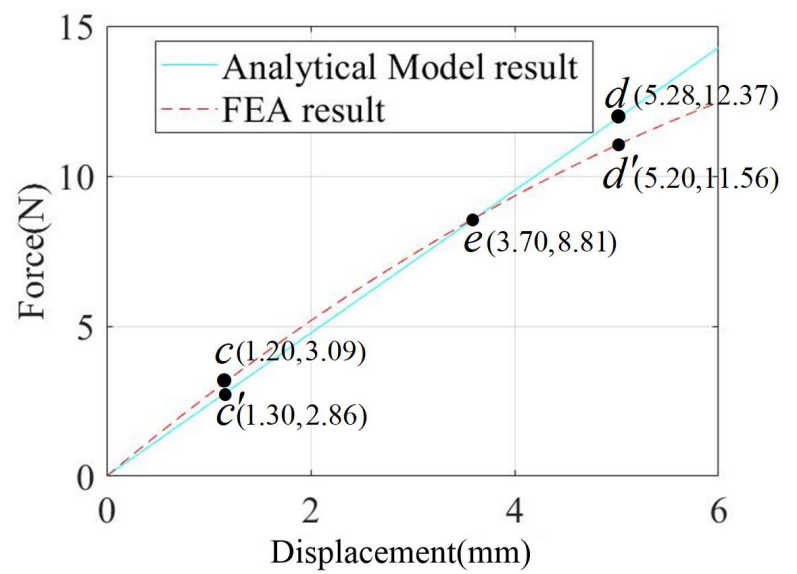

(b)

Fig. 6 FEA verification(a) theoretical and FEA results comparison of bistable beam, (b) theoretical and FEA results comparison of folded beam.

closely within the region of $[1.2-5.2] \mathrm{mm}$. The differences between the theoretical and FEA results are acceptable because of the established mathematic model ignores the effects of intermediate beams. In addition, the flatness of constant force travel range is poor, which is not suitable for the application requirements. Therefore, it is necessary to optimize architecture parameters to eliminate/reduce the force variation in the specified constant force range.

\section{Parameter Optimization}

The performance and dimensional size of the CFM is affected by the designed architecture parameters. For example, the parameter of inclination angle, length, and width of the CFM plays an important influence on the travel range of constant force. The design goal of CFM is taken as an end-effector which mounted at the end of 


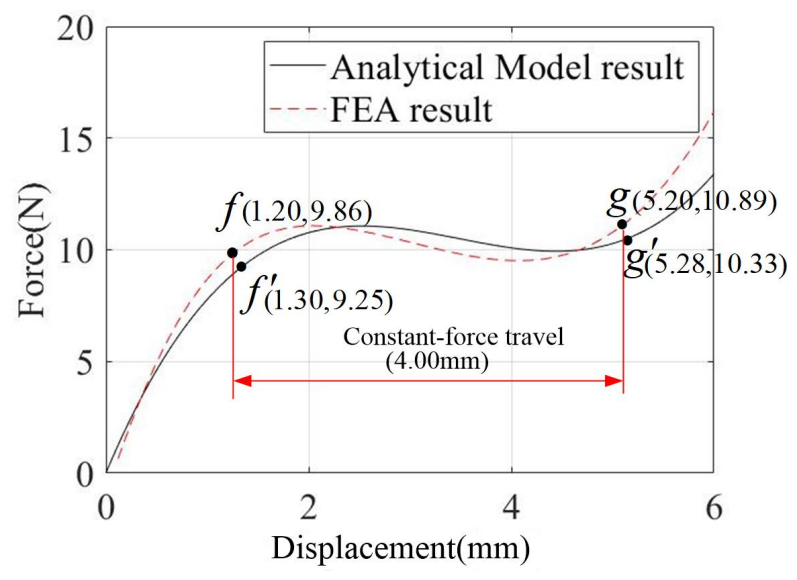

Fig. 7 Theoretical and FEA results comparison of the designed CFM.

robotic arm to implement polishing operation. Hence, the size compactness and mass of the CFM need to taken into consideration in terms of robotic loading capacity. In addition, considering the manufacturing method in this research, the width $d$ of the flexure beam is not less than $1 \mathrm{~mm}$. Because of the structural limitation, the length of $L$ is no longer than $40 \mathrm{~mm}$. The bigger inclination angle of the bistable beam, the larger negative stiffness displacement can generate, but the larger driving force required to generate buckling. To reduce the manufacturing error, the inclination angle should be larger than $4^{\circ}$. To eliminate force variation, the aforementioned design objectives and constraint conditions are summarized as follows:

Objective $: \min \sigma=\sqrt{\frac{1}{N} \sum_{1}^{N}\left(F_{x}-\mu\right)^{2}}$

Subject to $=\left\{\begin{array}{l}15 \leq L_{s 1} \leq 20 \\ 2 \leq L_{s 3} \leq 5 \\ 15 \leq L_{s 5} \leq 20 \\ 1 \leq b \leq 3 \\ 1 \leq d \leq 15 \\ 20 \leq L \leq 40 \\ 4^{\circ} \leq \theta \leq 7^{\circ}\end{array}\right.$

where $\sigma$ is the mean square error, $F_{x}$ represents the force value extracted in a given interval, and $\mu$ is the average force value of selected region. When the value of $\sigma$ is small or becomes zero, it means that force variation is reduced and eliminated.

Taking all the issues into account, PSO method is adopted to find the optimal solution of each parameter. In this research, the 200 sampled points are randomly selected in the range of $2.5 \mathrm{~mm}$ to $4.5 \mathrm{~mm}$. The obtained optimal parameters are listed in Tab.2. Substituting the optimal architecture parameters into the analytical model of CFM and conducting the simulation analysis, the FEA results are very consistent with analytical results as shown in Fig.8. It is further validated the correctness of the theoretical analysis. Referring to the Fig.8, the line located in the region of $2.5 \mathrm{~mm}$ to $4.5 \mathrm{~mm}$ is approximately a horizontal line, in which the maximum value is $12.63 \mathrm{~N}$, the minimum value is $12.43 N$ and the flatness is $98.41 \%$.

Table 2 Optimized architecture parameters of the CFM.

\begin{tabular}{lll}
\hline \hline parameter & value & unit \\
\hline $\mathrm{L}$ & 35.0 & $\mathrm{~mm}$ \\
$\mathrm{~b}$ & 2.0 & $\mathrm{~mm}$ \\
$\mathrm{~d}$ & 4.0 & $\mathrm{~mm}$ \\
$\theta$ & 5.1 & $\mathrm{deg}$ \\
$L_{s 1}$ & 20.0 & $\mathrm{~mm}$ \\
$L_{s 3}$ & 4.0 & $\mathrm{~mm}$ \\
$L_{s 5}$ & 19.0 & $\mathrm{~mm}$ \\
\hline \hline
\end{tabular}

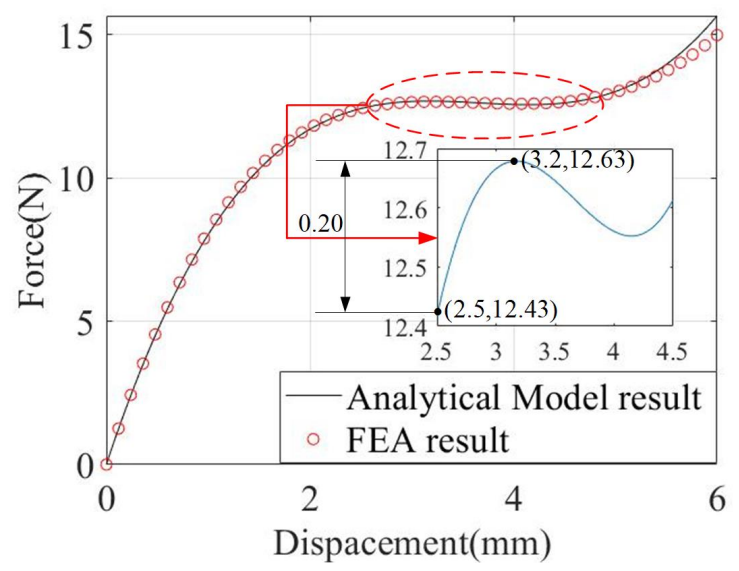

Fig. 8 Comparison of FEA simulation results and optimized results of the CFM.

\section{Experimental Verification}

To test the performance of the proposed CFM, the prototype is fabricated and a series of experimental studies are performed in this section.

\subsection{CFM fabrication}

To validate the proposed design, the experimental system is built as depicted in Fig. 9, including the designed CFM(fabricated by $3 \mathrm{D}$ printing), voice coil mo- 

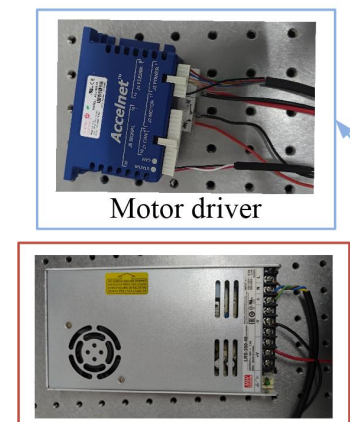

Motor drive power supply
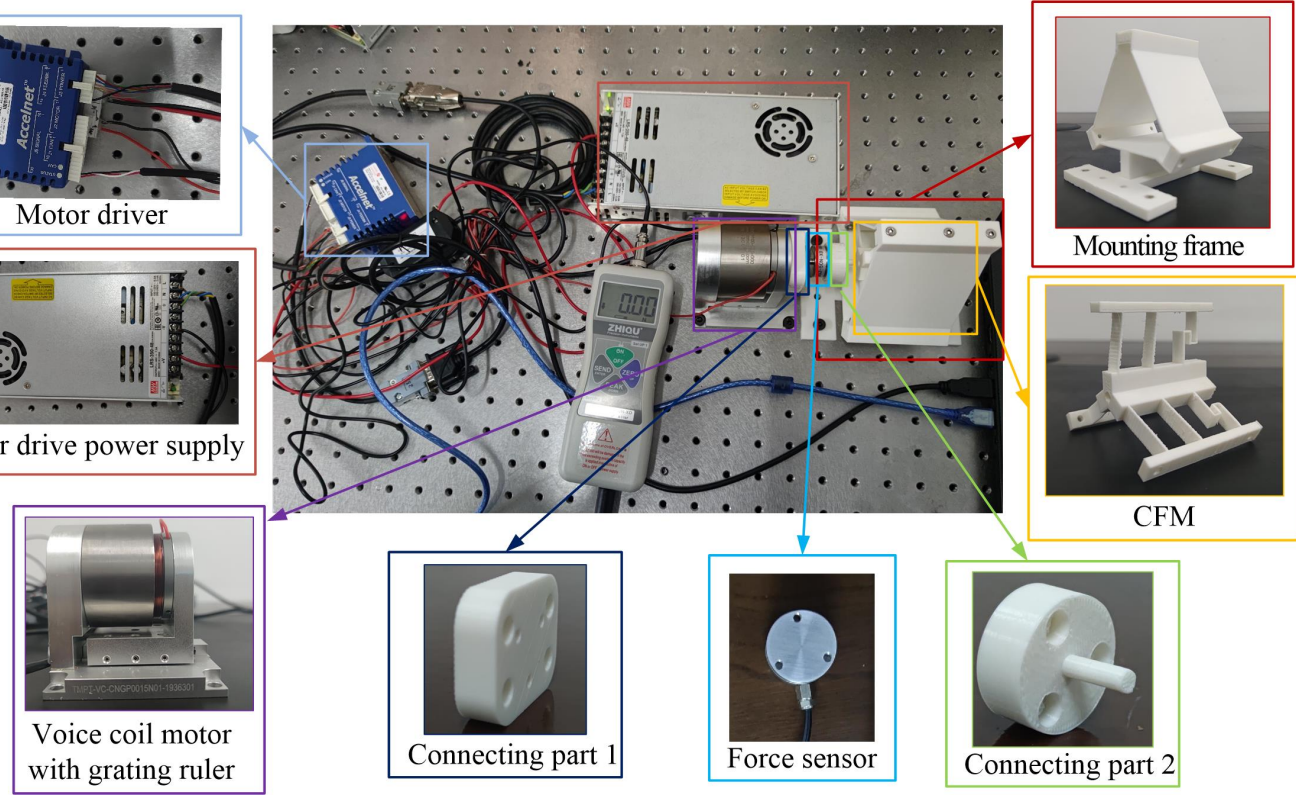

Fig. 9 Structure design of grinding mechanism with constant output force.

tor, grating ruler, force sensor, motor driver, and industrial control computer. To isolation the external disturbances from the environment, the whole experimental system is installed on an anti-vibration optical table. The commercial linear servo amplifier (model: ACJ055-18) is adopted to drive the voice coil motor (model: TMEC100-015-000) with a nominal travel range of 5 $m m$. A grating ruler (model: LaRW1-3D, Fagor Automation) is utilized to measure the displacement of the CFM. A force sensor (DS2-XD) with a range of $50 \mathrm{~N}$ and a resolution of $0.01 \mathrm{~N}$ is chosen to measure the reaction force.

\subsection{Relationship test between force and displacement}

During the experimental study, the actuating force and ouput displacement of the CFM are measured by the force sensor and grating ruler respectively. The comparison of FEA, theoretical calculation and experimental results is shown in Fig.10. Referring to the experimental results, the constant force range of the CFM starts from $2.68 \mathrm{~mm}$ to $4.81 \mathrm{~mm}$ with the constant force is $11.92 N$. Compared with the results of finite element analysis and theoretical calculation, the range of constant force increases by $0.18 \mathrm{~mm}$, while the size of constant force decreases by $0.68 \mathrm{~N}$.

\section{Conclusions}

This paper presents the design, modeling, simulation, optimization and experimental studies of a spa-

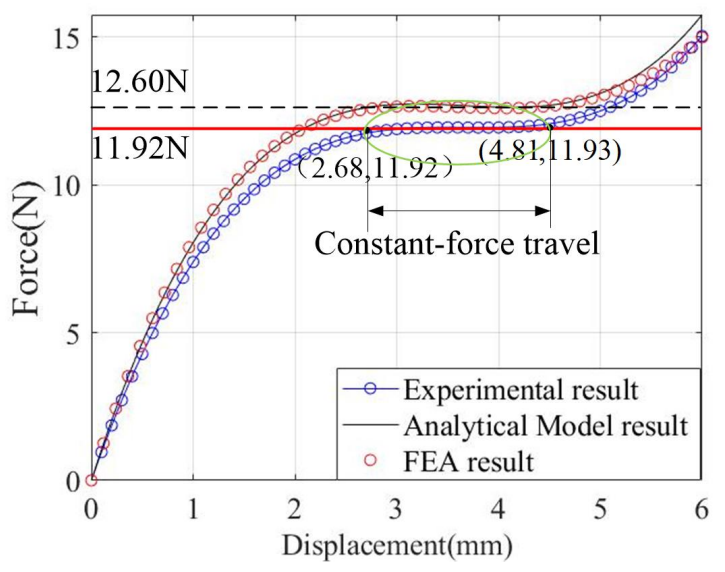

Fig. 10 Experimental results comparison with FEA simulation and analytical results.

tial CFM for potentially application in polishing operations. In order to enhance the mechanism stiffness and structure compactness, a parallel spatial CFM based on positive stiffness mechanism and negative stiffness mechanism is designed. To make full use of negative stiffness region, a novel folded beam mechanism with good linearity is designed and the force displacement relationship model referring to the PRB method is established. In addition, to eliminate the force variation, the PSO method is utilized to optimize the architectural parameters. According to the comparison of FEA results of optimized architecture and original design, the flatness in constant travel range is highly improved. Experimental results show that the proposed designed 
CFM possesses a good characteristic of output constant force.

\section{Fundings}

This work is supported by Huxiang High Level Talent Project of Hunan Province (Grant No. 2019RS1066), Education Department of Hunan Province (Grant No. 19C1520), the National Natural Science Foundation of China (Grant No. 51575544), the General Research Fund of the Research Grants Council(RGC) of Hong Kong, China (Grant No. PolyU 152137/19E), and the Research Committee of Hong Kong Polytechnic University (Grant No.1-45-37-ZE97).

\section{Conflicts of Interest}

The authors declare no conflict of interest.

\section{Author contributions}

Bingxiao Ding provided the concept of the work, built up the model, Jiyu Zhao designed mechanism and conducted experimental verification, and Yangmin Li carried out supervision and revision of this paper.

\section{Availability of data and material}

The data of this study are available from the first author or corresponding author upon reasonable request.

\section{References}

Wan, S, Zhang, X, Wang, W et al. Effect of pad wear on tool influence function in robotic polishing of large optics. International Journal of Advanced Manufacturing Technology, 2019, 102, 2521-2530.

Tao B, Zhao X, Ding H, Mobile-robotic machining for large complex components: a review study. Science ChinaTechnological Sciences, 2019, 62(8) 1388-1400.

Mohammad A E K, Wang D, A novel mechatronics design of an electrochemical mechanical end-effector for roboticbased surface polishing, 2015 IEEE/SICE International Symposium on System Integration (SII), 2015, pp. 127133.

Domroes F, Krewet C, Kuhlenkoetter B. Application and Analysis of Force Control Strategies to Deburring and Grinding. Modern Mechanical Engineering, 2013, 3(2A): 11-18.

Zhu D H, Xu X H, Yang Z Y, et al. Analysis and assessment of robotic belt grinding mechanisms by force modeling and force control experiments. Tribology International, 2018, 120: 93-98.
Kim U, Lee D H, Kim Y B, et al. A novel 6-axis force/torque sensor for robotic applications. IEEE/ASME Transaction on Mechatronics, 2017;22(3):1381-91.

Budak E. Improving Productivity and Part Quality in Milling of litanium Based Impellers by Chatter Suppression and Force Control. Annals of the ClRP, 2000, 49(1):31-36

Tian, F, Li, Z, Lv, C et al. Polishing pressure investigations of robot automatic polishing on curved surfaces. International Journal of Advanced Manufacturing Technology, 2016, 87, 639-646.

Lopes A, Almeida F. A force-impedance controlled industrial robot using an active robotic auxiliary device. Robotics and Computer Integrated Manufacturing, 2008,24:299309

Solanes J E, Gracia L, Benavent P M, et al. Adaptive robust control and admittance control for contact-driven robotic surface conditioning, Robotics and Computer Integrated Manufacturing.,2018,54: 115-132

Xian J S, Xi Z, et. al. Fuzzy adaptive hybrid impedance control for mirror milling system, Mechatronics, 2018, 53: 20-27

Latifinavid M, Donder A, Konukseven E I, High-performance parallel hexapod-robotic light abrasive grinding using real-time tool deflection compensation and constant resultant force control. The International Journal of Advanced Manufacturing Technology, 2018, 96.9-12: 34033416

Wang Q L, Wang W, Zheng Y L, et al. Force control-based vibration suppression in robotic grinding of large thin-wall shells. Robotics and Computer Integrated Manufacturing. 2021,67:1-12.

Xie Q L, Zhao H, Wang T, et al. Adaptive Impedance Control for Robotic Polishing with an Intelligent Digital Compliant Grinder. International Conference on Intelligent Robotics and Applications, 2019, 482-484.

Xu X H, Zhu D H, Zhang H Y, et. al. Application of novel force control strategies to enhance robotic abrasive belt grinding quality of aero-engine blades. Chinese Journal of Aeronautics, 2019, 32(10): 2368-2382.

Du H, Sun Y, Feng D, et al. Automatic robotic polishing on titanium alloy parts with compliant force/position control, Proceedings of the Institution of Mechanical Engineers, Part B: Journal of Engineering Manufacture,2015,7: 1180-1192

Liao L, Xi F F, Liu K F. Modeling and control of automated polishing/deburring process using a dual-purpose compliant toolhead, International Journal of Machine Tools and Manufacture, 2008, 48(12-13): 1454-1463

Satake U, Enomoto T, Obayashi Y, et al. Reducing edge rolloff during polishing of substrates, Precision Engineering, 2018, 51: 97-102

Mohammad A E K, Hong J, Wang D W, et al. Design of a force-controlled end-effector with low-inertia effect for robotic polishing using macro-mini robot approach. Robotics and Computer-Integrated Manufacturing, 2018, 49: 54-65.

Chen F, Zhao H, Li D W, et al. Contact force control and vibration suppression in robotic polishing with a smart end effector. Robotics and Computer-Integrated Manufacturing, 2019, 57: 391-403.

Liu X, Zhang T, Li J, et al. A Novel End-effector for Robotic Compliant Polishing. IEEE International Conference on Robotics and Biomimetics. 2018. 1858-1863.

Tang J, Wang T, Yan Z Q, et al. Design and Analysis of the End-effector Of the Flexible Polishing Robot. Key Engineering Materials,2016, 693: 58-63. 
1 Roughness in Robotic Grinding Process, ROMANSY 22, Robot Design, Dynamics and Control. Springer, 2019:363-369

Holst G L, Teichert G H, Jensen B D. Modeling and Experiments of Buckling Modes and Deflection of Fixed-Guided Beams in Compliant Mechanisms. Journal of Mechanical Design,2011,133: 051002-1.

Zhao J, Jia j y, He X B, et al. Post-buckling and SnapThrough Behavior of Inclined Slender Beams. Journal of Applied Mechanics,2008,75: 041020-1.

Chi W W, Azid A A, Majlis B Y. Formulation of stiffness constant and effective mass for a folded beam. Archives of Mechanics, 2010, 62(5): 405-418. 
Figures

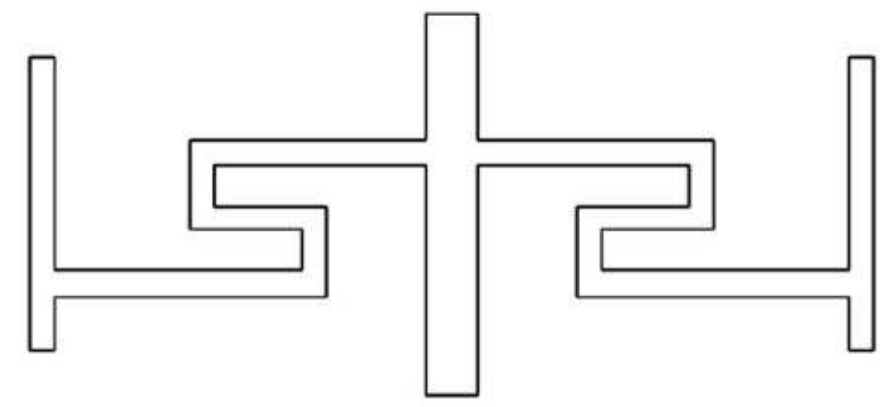

Folded beam model

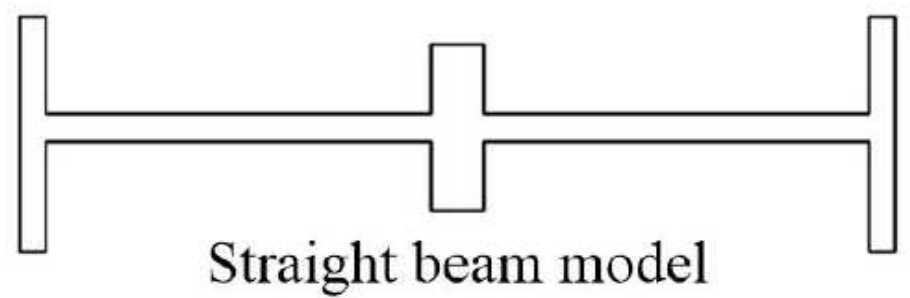

(a)

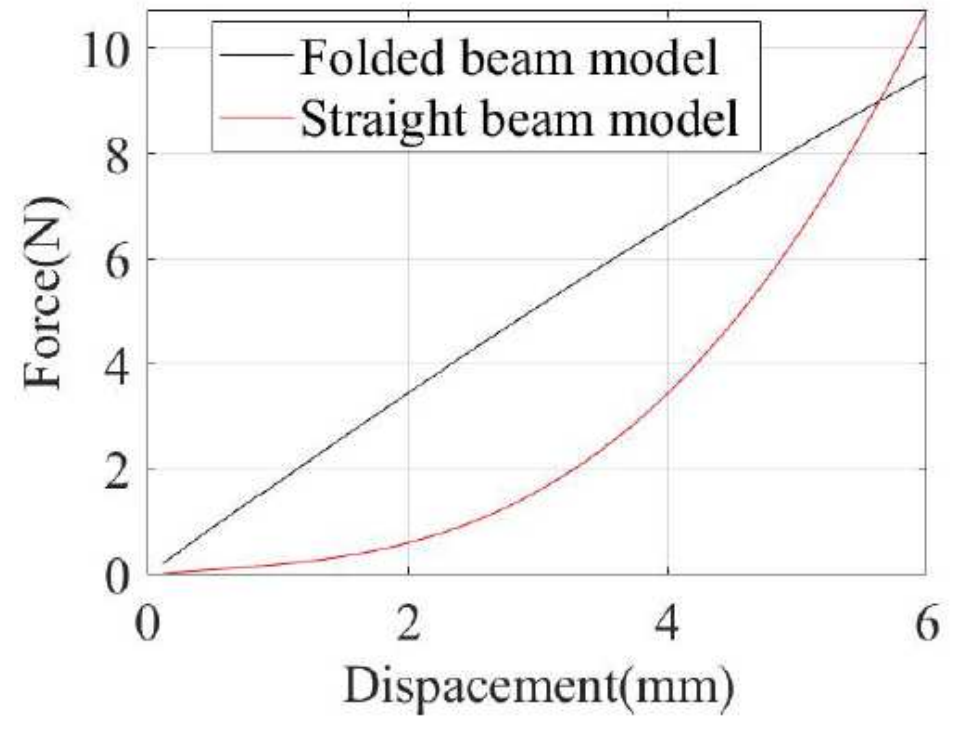

(b)

Figure 1

Comparison of two kinds of positive stiffness mechanism.

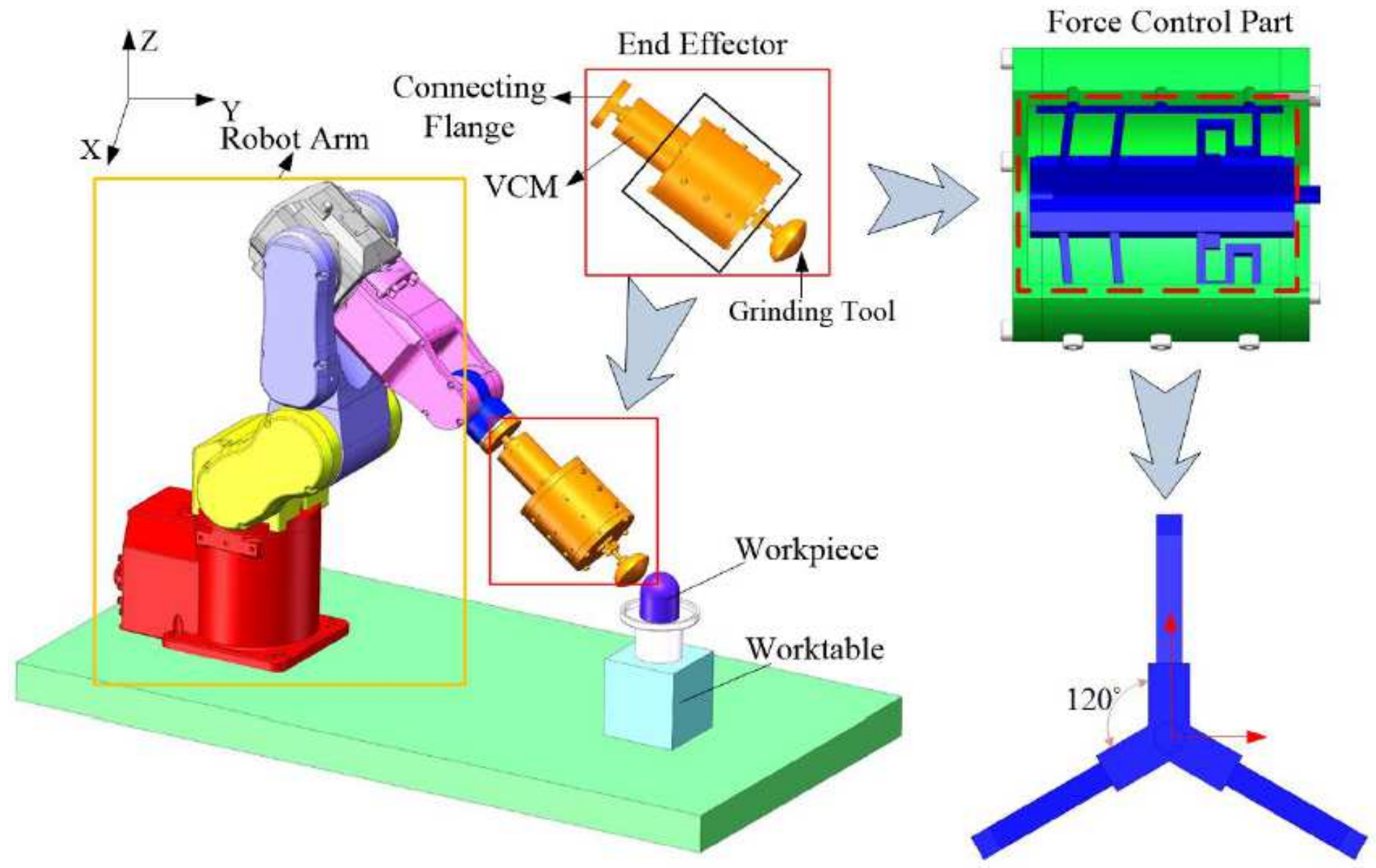


Figure 2

Structure design of grinding mechanism with constant output force.

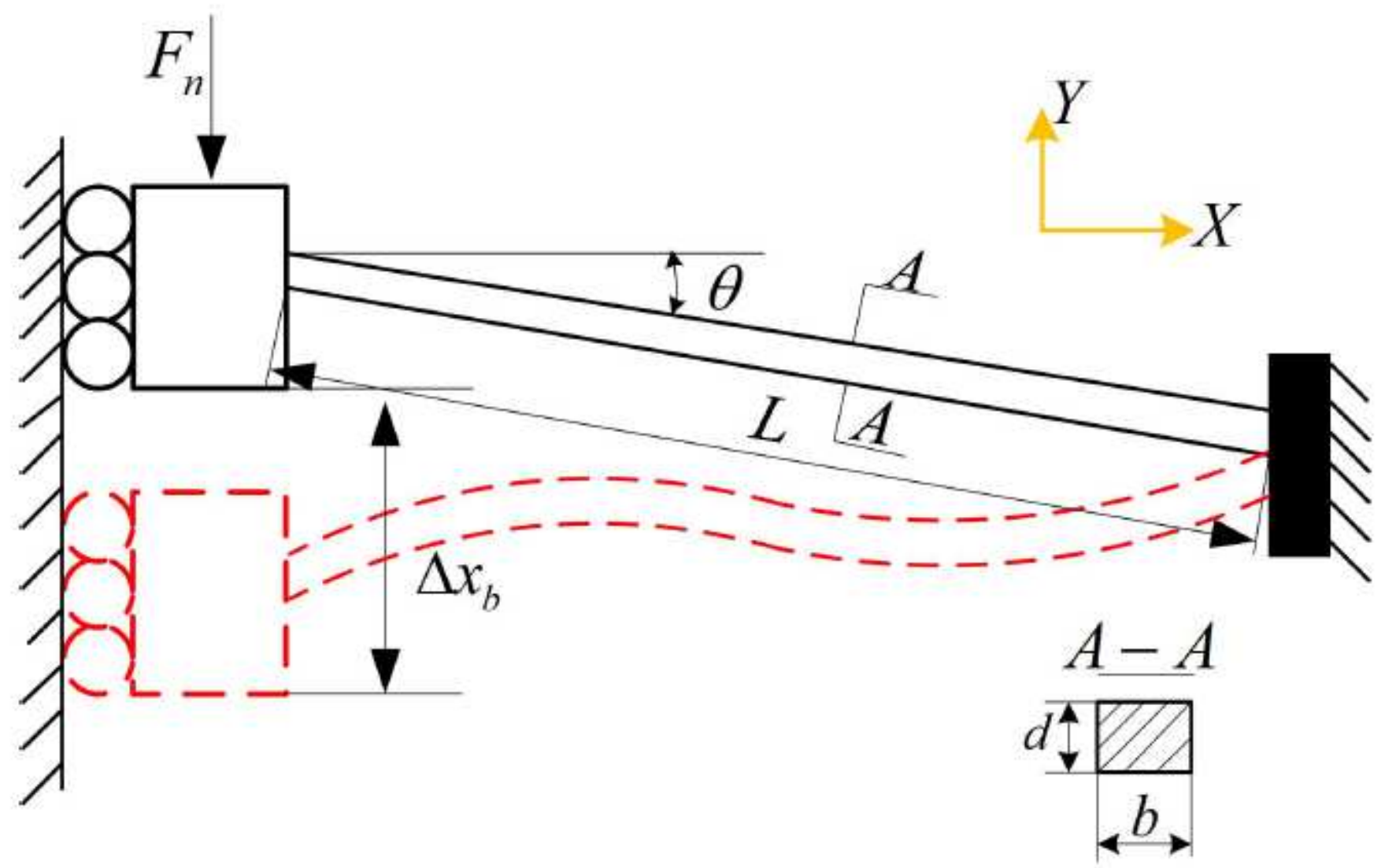

Figure 3

Buckling deformation of bistable beam mechanism. 


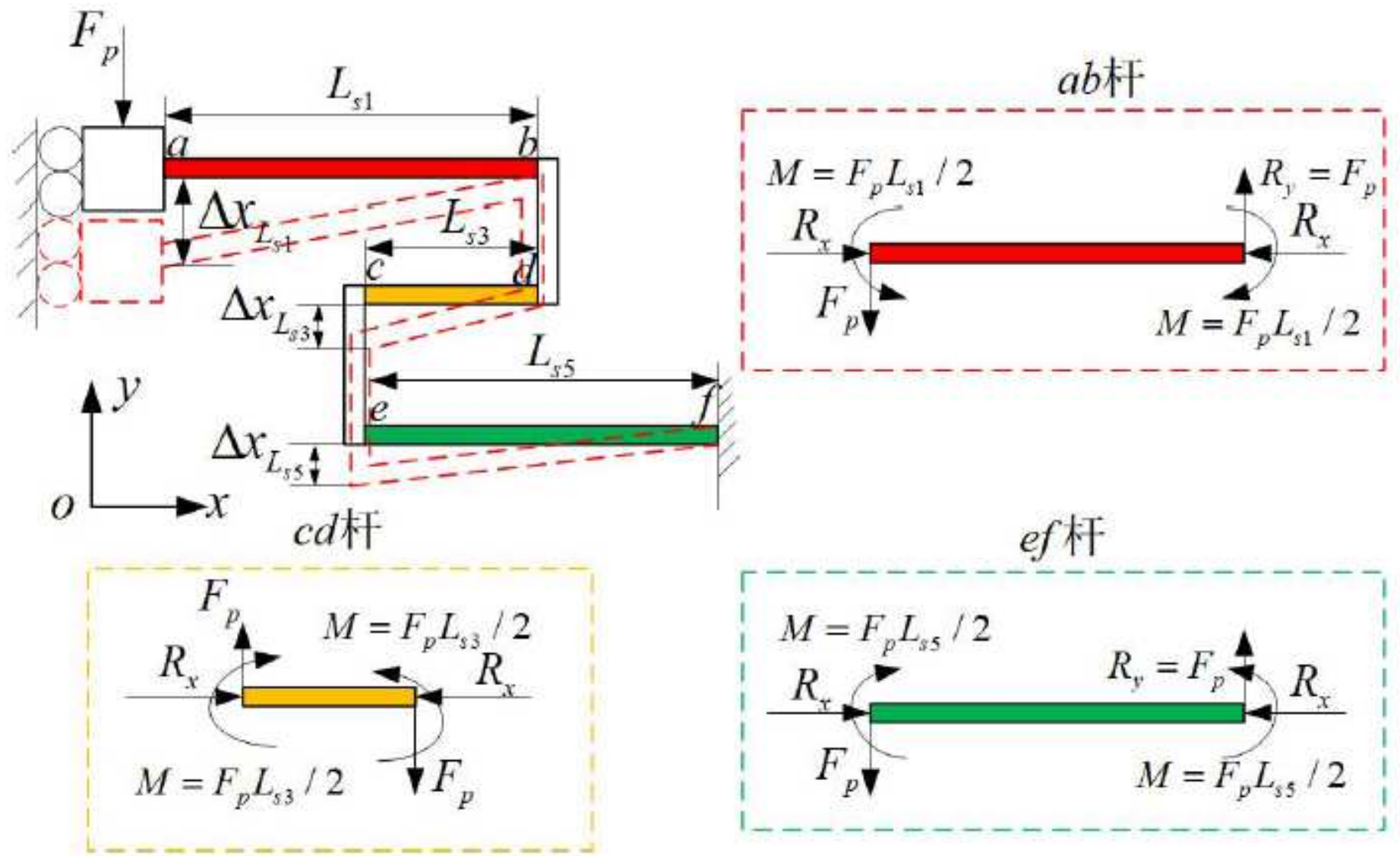

Figure 4

Resolved components of folded beam.

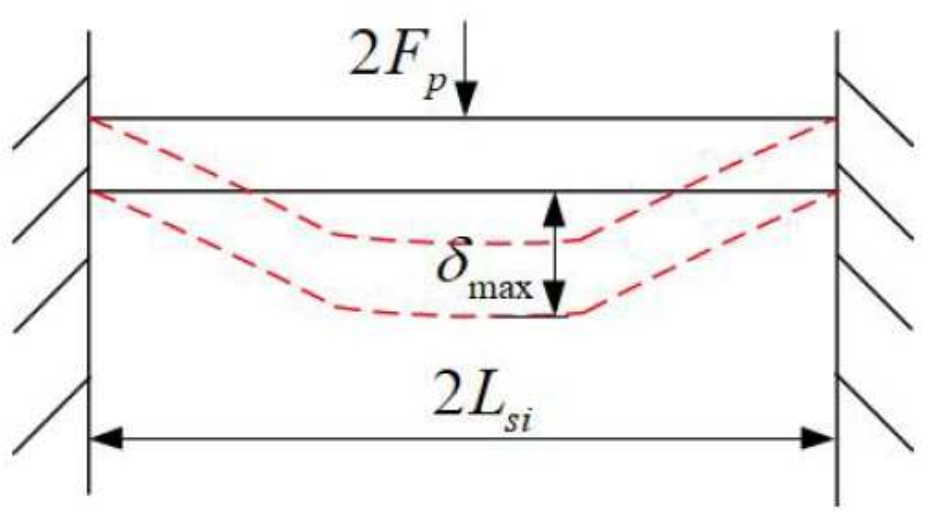

(a)

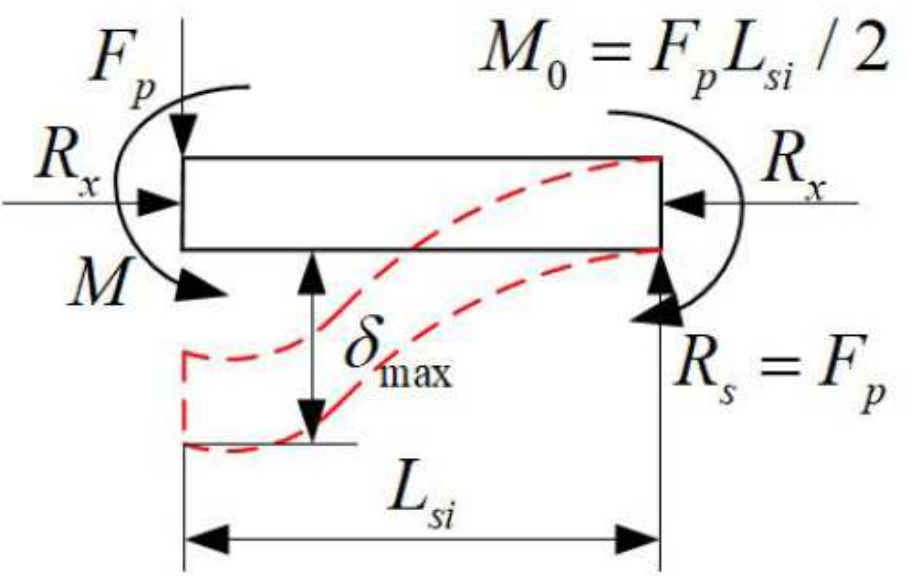

(b)

Figure 5

(a) fixed fixed beam under transverse loading (b) the model of half fixed beam 


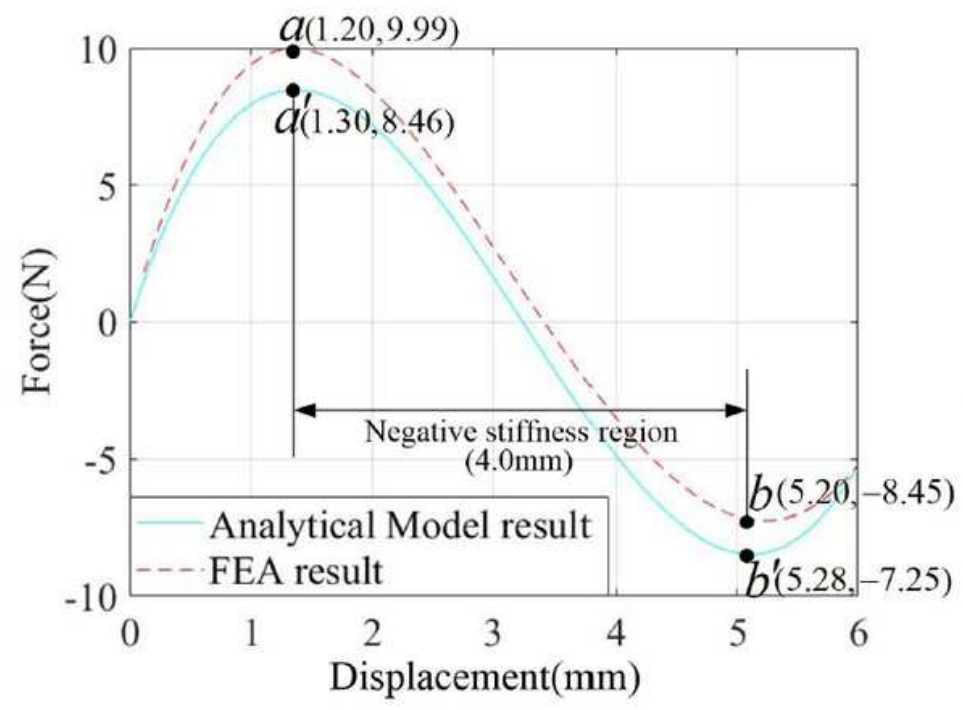

(a)

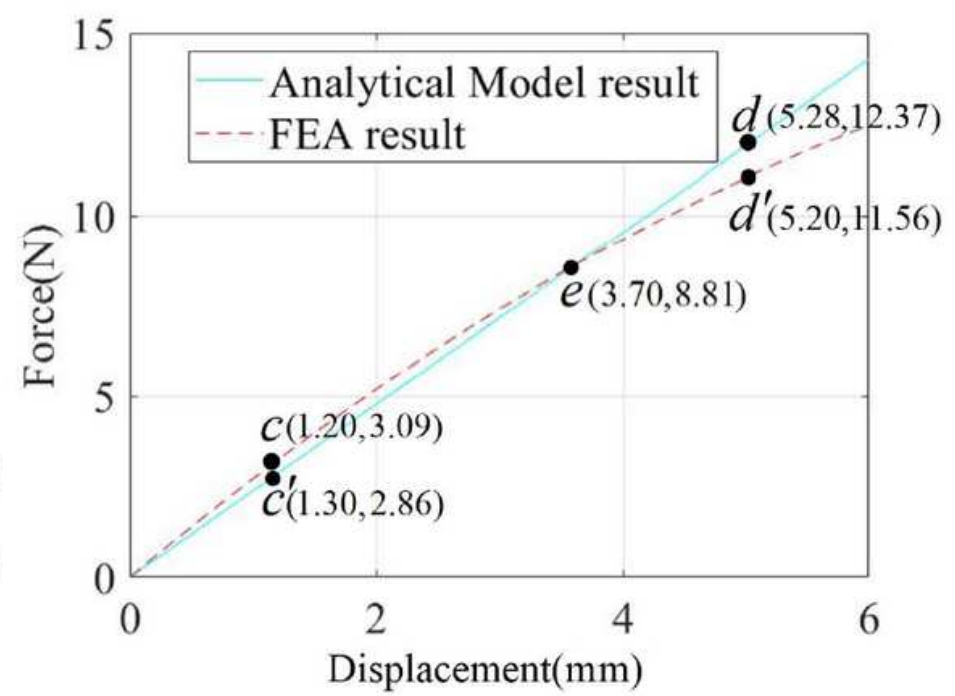

(b)

Figure 6

FEA verication(a) theoretical and FEA results comparison of bistable beam, (b) theoretical and FEA result comparison of folded beam.

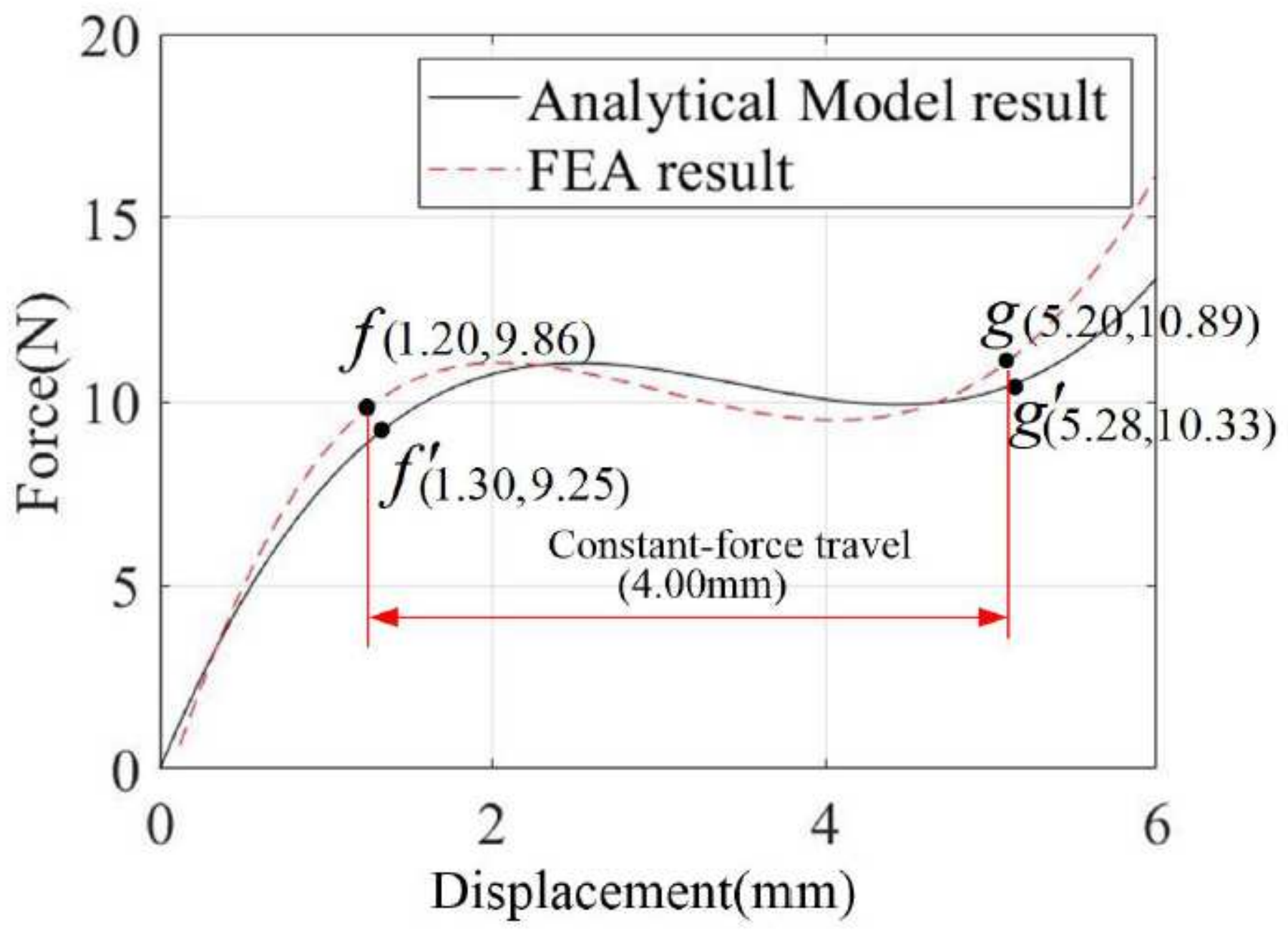


Figure 7

Theoretical and FEA results comparison of the designed CFM.

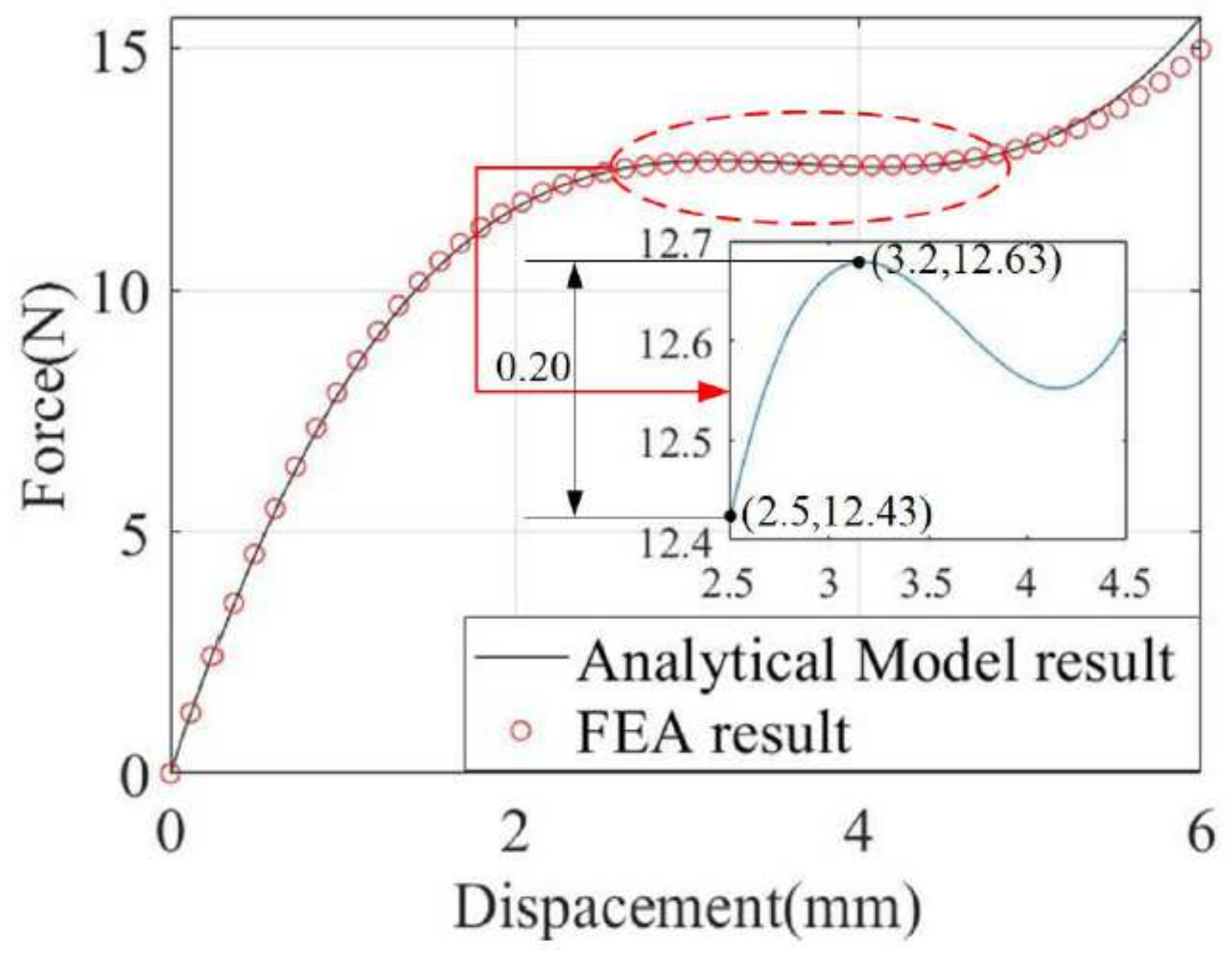

Figure 8

Comparison of FEA simulation results and optimized results of the CFM. 


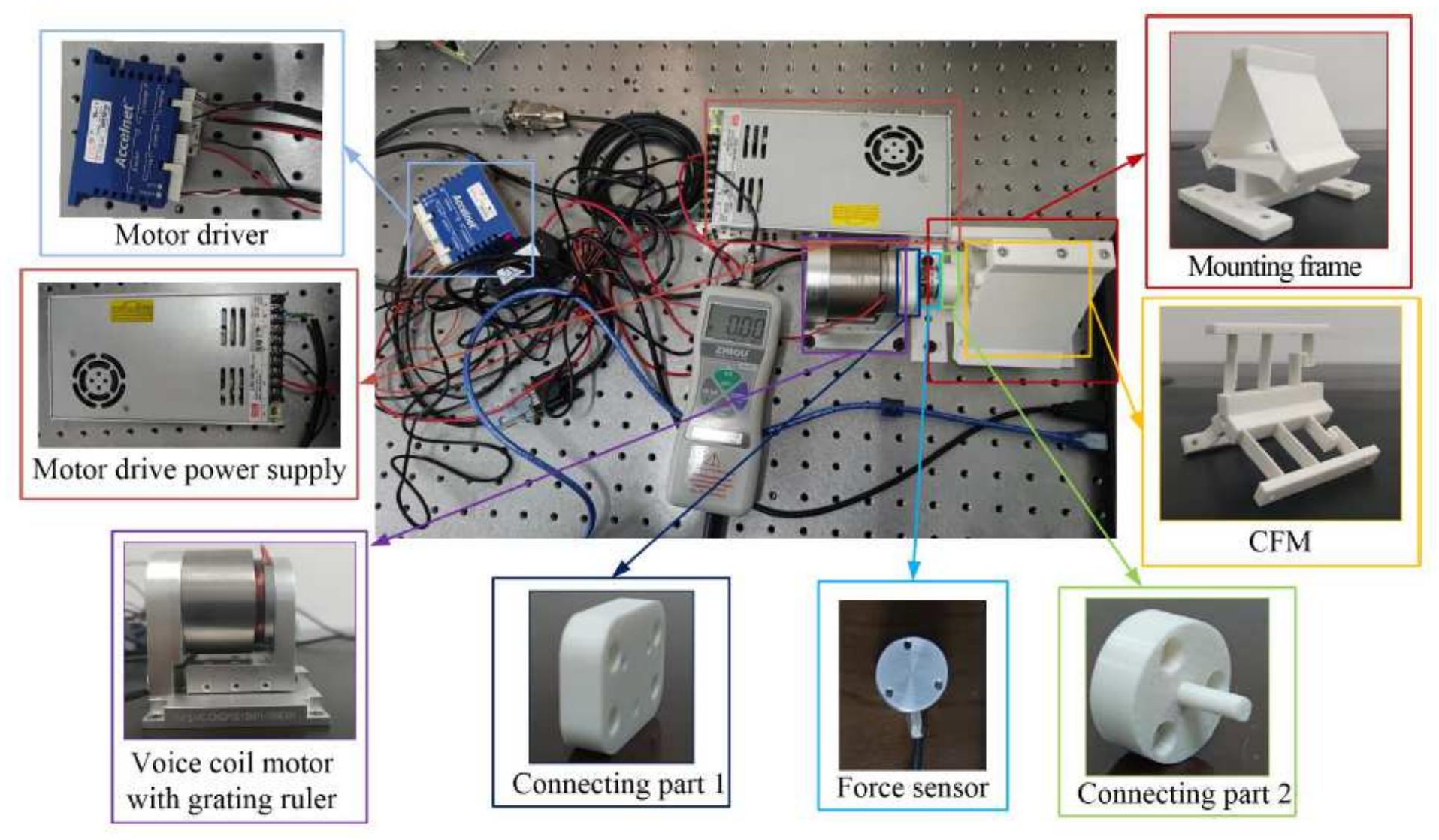

Figure 9

Structure design of grinding mechanism with constant output force. 


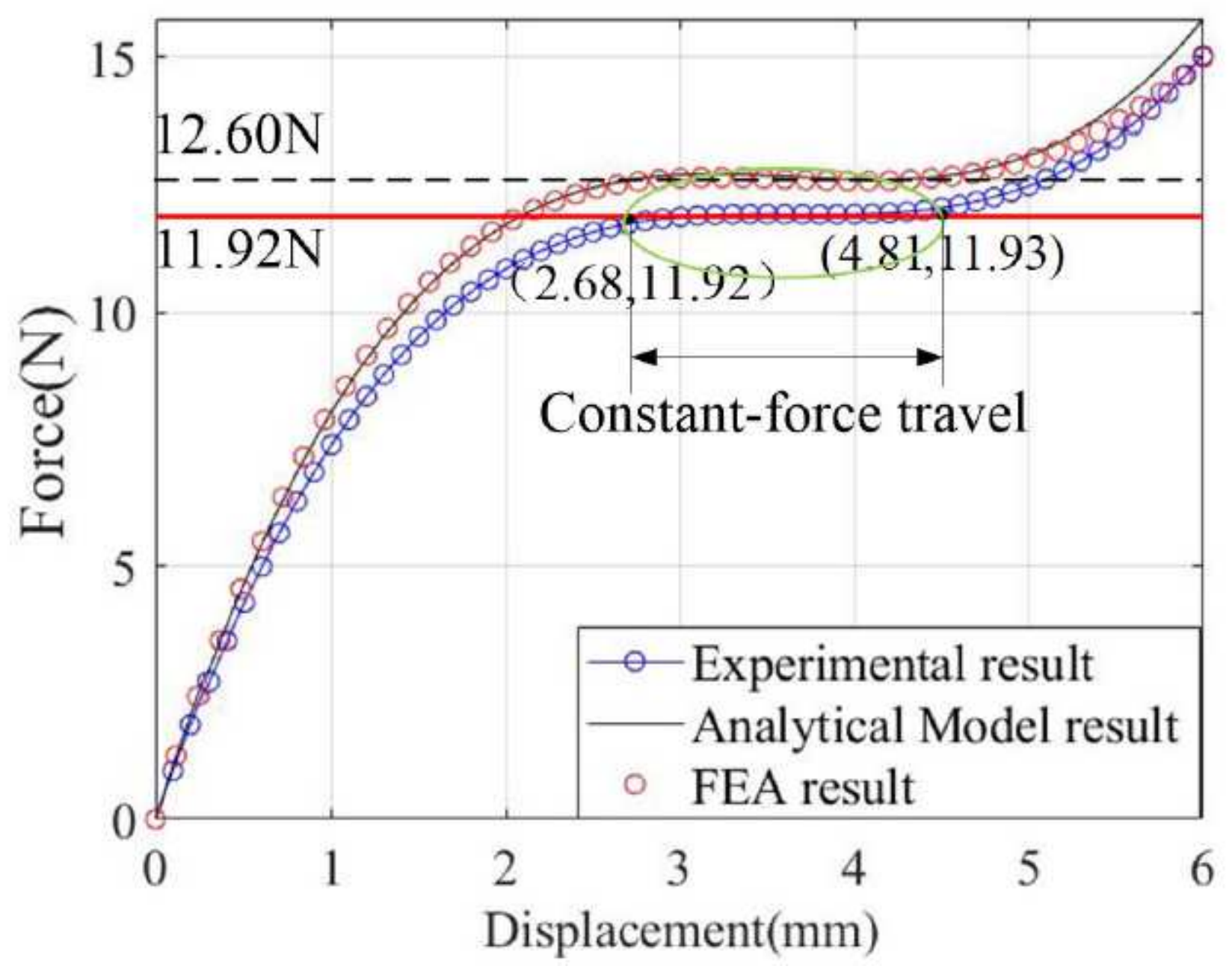

Figure 10

Experimental results comparison with FEA simulation and analytical results. 